\title{
The genus Parasola: Phylogeny and the description of three new species
}

\section{János G. Szarkándi, Geert Schmidt-Stohn, Bálint Dima, Shah Hussain, Sándor} Kocsubé, Tamás Papp, Csaba Vágvölgyi \& László G. Nagy

To cite this article: János G. Szarkándi, Geert Schmidt-Stohn, Bálint Dima, Shah Hussain, Sándor Kocsubé, Tamás Papp, Csaba Vágvölgyi \& László G. Nagy (2017): The genus Parasola: Phylogeny and the description of three new species, Mycologia

To link to this article: http://dx.doi.org/10.1080/00275514.2017.1386526

Accepted author version posted online: 09 Oct 2017.

Submit your article to this journal

LII Article views: 9

Q View related articles $\sqsubset$

View Crossmark data ‘ 


\title{
The genus Parasola: Phylogeny and the description of three new
}

\section{species}

\author{
János G. Szarkándi \\ Közép fasor 52, H-6726 Szeged, Hungary \\ szarkandi.g@gmail.com \\ Geert Schmidt-Stohn \\ Burgstrasse 25, D-29553 Bienenbüttel, Germany \\ geert.schmidt-stohn@t-online.de
}

Department of Microbiology, University of Szeged, Faculty of Science and Informatics,

\section{Bálint Dima}

Department of Plant Anatomy, Institute of Biology, Eötvös Loránd University

Pázmány Péter sétány 1/C, H-1117 Budapest, Hungary

Department of Biosciences (Plant Biology), Viikki Plant Science Centre, University of Helsinki, P.O. Box 65, FI-00014, Helsinki, Finland

cortinarius1@gmail.com

Shah Hussain

Center for Plant Sciences and Biodiversity, University of Swat, Mingora 19130, Pakistan

shahpk85@gmail.com

\section{Sándor Kocsubé}

Department of Microbiology, University of Szeged, Faculty of Science and Informatics, Közép fasor 52, H-6726 Szeged, Hungary

shigsanyi@gmail.com 
Tamás Papp

MTA-SZTE "Lendület" Fungal Pathogenicity Mechanisms Research Group, Közép fasor 52, H-6726 Szeged, Hungary

pappt71@gmail.com

\section{Csaba Vágvölgyi}

Department of Microbiology, University of Szeged, Faculty of Science and Informatics, Közép fasor 52, H-6726 Szeged, Hungary

mucor1959@gmail.com

László G. Nagy ${ }^{1}$

Synthetic and Systems Biology Unit, Institute of Biochemistry, BRC, HAS, Temesvári körút 62, H-6726 Szeged, Hungary

Corresponding author: László G. Nagy, lnagy@fungenomelab.com

Received 15 November 2016

Accepted 27 September 2017

\section{ABSTRACT}

Parasola represents an enigmatic lineage of veil-less, coprinoid fungi in Psathyrellaceae (Agaricales). The species-level taxonomy of the genus has been in a flux recently, resulting in the elimination of some long-established names and the description of new taxa. Here, we reconstruct the phylogeny of Parasola using two nuc rDNA loci, the internal transcribed spacers region (ITS1-5.8S-ITS2) and 28S and identify several putatively undescribed species, of which three are formally described here (Parasola crataegi, P. ochracea and P. plicatilissimilis) based on molecular and morphological data. Morphological descriptions for the new 
species and an identification key to accepted Parasola species are given. We revise and discuss our current understanding of the phylogeny of Parasola.

KEY WORDS: coprinoid fungi, generic phylogeny, nuc rDNA, revision, species description

Phylogeny of Parasola

Szarkándi et al.: Phylogeny of Parasola

\section{INTRODUCTION}

Parasola Redhead, Vilgalys \& Hopple is a genus of small, coprinoid mushrooms with colors ranging from ochre-orange to lilac with most being brownish, belonging to Psathyrellaceae Vilgalys, Moncalvo \& Redhead (Redhead et al. 2001; Nagy et al. 2009). The species are common saprotrophic agarics found on lawns, along forest trails and in grassland habitats with a few species growing on dung, e.g. Parasola misera (P. Karst.) Redhead, Vilgalys \& Hopple, P. cuniculorum D.J. Schaf. (Schafer 2014), or occasionally P. conopilus (Fr.) Örstadius \& E. Larss. (Padamsee et al. 2008; Larsson \& Örstadius 2008; Schafer 2010, 2014).

Parasola species have small and deeply grooved parasol or umbrella-like pilei, ranging from vivid orange-ochraceous to lilac but are mostly yellow-brown to tawny and paler colors. Parasola differs from the closely related Coprinellus P. Karst, Coprinopsis P. Karst. and Psathyrella (Fr.) Quél. in the complete absence of a veil structure and the lack of pileo- and caulocystidia, although P. conopilus, P. auricoma (Pat.) Redhead, Vilgalys \& Hopple, P. setulosa (Berk. \& Broome) Redhead, Vilgalys \& Hopple, and P. malakandensis S. Hussain, N. Afshan \& H. Ahmad possess sclerocystidia (Nagy et al. 2010a). Basidiocarps of Parasola are non-deliquescent, but show some level of tissue degradation (Buller 1931; 
Redhead et al. 2001; Larsson \& Örstadius 2008) referred to as incomplete deliquescence or collapse (Nagy et al. 2009, 2012). Basidiospores are dark, generally rounded triangular, heart-shaped or ovoid with a mostly eccentric germ pore, although earlier-diverging species tend to have ellipsoidal basidiospores. Molecular phylogenetic analyses recover the monophyly of Parasola with strong support (Padamsee et al. 2008; Nagy et al. 2009, 2010b, 2012).

The genus is divided into two sections by the presence or absence of thick-walled, brown sclerocystidia (setae) on their pilei (Schafer 2010). Section Auricomi (Singer) D.J. Schaf. has such setae and section Parasola does not. According to Index Fungorum (www.indexfungorum.org) the genus currently has 22 species, although recent taxonomic treatments report a lower number of recognized taxa (Nagy et al. 2009, 2010a; Schafer 2010, 2014). This number has fluctuated because of synonymizations, e.g. P. leiocephala (P.D. Orton) Redhead, Vilgalys \& Hopple with P. lactea (A.H. Sm.) Redhead, Vilgalys \& (Nagy et al. 2010a) and because of the discovery of new species. Recently, two new species were described, Parasola cuniculorum, differing from P. misera (P. Karst.) Redhead, Vilgalys \& Hopple by having two-spored basidia (Schafer 2014), and P. malakandensis, closely related to P. setulosa (Berk. \& Broome) Redhead, Vilgalys \& Hopple (Hussain et al. 2017).

In this study, we describe three new species in Parasola based on an extended sampling of specimens for phylogenetic analyses. Species descriptions are based on morphological characters and molecular phylogenetic analyses of the internal transcribed spacer regions (ITS1-5.8S-ITS2 $=$ ITS) and the D1-D2 domains of the $28 \mathrm{~S}$ gene of the nuc rDNA (28S). We provide an identification key for currently accepted species of Parasola, including the three newly described species and other putatively new species. 


\section{MATERIALS AND METHODS}

Taxon sampling, laboratory protocols.-We sampled collections of 15 currently accepted species in Parasola, and collections of 7 additional apparently undescribed species, selected on the basis of morphological observations and preliminary sequence data. We extracted genomic DNA from dried basidiomes using the DNeasy Plant Mini Kit (Qiagen, Redwood City, California, USA) and amplified the nuclear ribosomal loci using primers LROR and LR7 for the 28S gene, and ITS1 and ITS4 for the ITS region (White et al. 1990). Standard PCR protocols were used for the amplification using DreamTaq polymerase (Thermo Scientific, Waltham, Massachusetts, USA) (White et al. 1990; Gardes \& Bruns 1993). PCR products were purified and sequenced by LGC Genomics Ltd (Berlin, Germany) in both directions and reads were assembled into contigs by using CodonCode Aligner (CodonCode Corporation, Centerville, Massachusetts, USA) and DNA Baser (Heracle BioSoft SRL, Lilienthal, Germany). All sequence accession numbers produced for this study (KY928603KY928647) are shown in Supplementary Table 1. Contigs were evaluated by BLAST against NCBI's nucleotide database (Altschul et al. 1990). Additional 28S and ITS sequences were obtained from GenBank for outgroup species (Supplementary Table 1).

Alignments and phylogenetic reconstruction.-We aligned $28 \mathrm{~S}$ and ITS sequences independently using PRANK 091016 (Löytynoja \& Goldman 2005) with default settings and corrected the alignments manually after visual inspection in JALVIEW 2.4.0 (Waterhouse et al. 2009). During inspection we discarded sequences shorter than $1000 \mathrm{bps}$ for $28 \mathrm{~S}$ and $400 \mathrm{bps}$ for ITS. We concatenated the ITS and 28S alignments into a supermatrix. Indels in the ITS region were recoded as presence/absence characters using the simple indel coding algorithm (Simmons \& Ochoterena 2000) as implemented in gapcode.py (version 2.1, http://www.bioinformatics.org/ rick/software.html). For Bayesian analyses this matrix was 
added to the concatenated alignment. We also analyzed the ITS and $28 \mathrm{~S}$ alignments independently. We chose Coprinopsis lagopus (Fr.) Redhead, Vilgalys \& Moncalvo, C. picacea (Bull.) Redhead, Vilgalys \& Moncalvo, C. pseudonivea (Bender \& Uljé) Redhead, Vilgalys \& Moncalvo and C. marcescibilis (Britzelm.) Örstadius \& E. Larss. as outgroups.

Phylogenetic inference was conducted using Bayesian and Maximum Likelihood (ML) methods for both the concatenated and the independent ITS and 28S datasets. For Bayesian inference, we used MRBAYES 3.2.1 (Ronquist et al. 2012) with a partitioned GTR+ $\Gamma$ model, a chain length of 10 million generations, sampling frequency 100 , two independent replicates with 3 heated and one cold chains per replicate. We partitioned the concatenated alignment into ITS, $28 \mathrm{~S}$ and indel matrices and the parameters of the evolutionary model were estimated separately for the individual partitions. Indels were modeled by a 2 -state Markov model for restriction sites implemented in MRBAYES. We established burn-in by inspecting the convergence of likelihood values in Tracer 1.6 (Rambaut et al. 2014). Post burn-in trees were used to compute a $50 \%$ majority rule consensus tree using the SUMTREES script of the DENDROPY package (Sukumaran \& Holder 2010). Maximum Likelihood analyses were run in RAXML 7.0.4 (Stamatakis 2006) under the GTR $+\Gamma$ model and two partitions (ITS and 28S). Branch support was calculated by 500 non-parametric bootstrap replicates and mapped onto the ML tree using SUMTREES. Nodes were considered strongly supported when bootstrap values (BS) were $\geq 70 \%$ and posterior probability values (PP) were $\geq 0.95$.

Constraint analyses were performed using the CONSEL package (Shimodaira \& Hasegawa 2001) on the concatenated dataset. Topological constraints were designed by hand in Mesquite 2.75 (Maddison \& Maddison 2011), and used in RAXML to estimate site-wise likelihoods. In RAXML we inferred ten constrained and 10 unconstrained trees, which were then compared using the approximately unbiased test (at $\mathrm{p}<0.05)$. Datasets were submitted to 
TreeBase (TB2:S20867).

Morphological descriptions. - Line drawings of microscopic characters are based on microphotographs; basidiospores were partly drawn with a microscope-adapter. Measurements were made at $1000 \times$ magnification with a calibrated optical micrometer. Basidiospore measurements are based on at least 20 basidiospores from each collection; numbers in square brackets refer to the number of basidiospores measured, the number of basidiocarps and the collections they originate from, respectively. Spore measurements are given as follows: length range $\times$ breadth range $\times$ width range. $Q_{1}$ and $Q_{2}$ values were calculated as follows: $\mathrm{Q}_{1}=$ length divided by breadth; $\mathrm{Q}_{2}=$ length divided by width. Measurements of basidia included sterigmata. The calculation of the proportion of 4-, 3- or 2spored basidia was made according to the methods of C. Bas (pers. comm.) and SchmidtStohn (2012): an even, ripe piece of lamella is placed on a piece of moist paper tissue on a microscope slide. The basidia are then observed on the face of the lamella with $40 \times$ or $400 \times$ magnification and the number of basidiospores per basidium is counted. Pleurocystidia and cheilocystidia were observed and measured by cutting the lamellae edge from the rest of lamella to avoid blending of the two types of cystidia.

\section{RESULTS}

Phylogenetic analyses.-We generated 28 new sequences from 12 species. The concatenated alignment comprised 77 specimens with 2166 nucleic acid sites (ITS: 785 sites, 28S: 1381 sites) and 167 binary characters coded from indels in the ITS alignment. The ITS alignment comprised 77 specimens with 785 nucleic acid sites and the $28 \mathrm{~S}$ alignment consisted of 58 specimens with 1381 nucleic acid sites. Phylogenetic trees reconstructed using ML and Bayesian methods were largely congruent with each other and reflected current views on the 
phylogeny of the genus (FIG. 1., SuPPlementary Figs 1-4. Nagy et al. 2009). Briefly, both inference strategies recover Parasola conopilus, $P$. auricoma, P. setulosa and $P$. malakandensis as basal groups with strong support and grouping species of section Parasola in a 'crown' Parasola clade. Parasola malakandensis (Hussain et al. 2017) grouped closely to the crown-group of Parasola (1.00/81) occupying an intermediate position between species of section Auricomi and those of section Parasola. The phylogeny indicated three species-level groups that could not be assigned to known species, and are described here as $P$. crataegi, P. ochracea and P. plicatilis-similis. Parasola crataegi was inferred basal to a clade formed by $P$. misera and P. lactea. Parasola plicatilis-similis was inferred sister to the clade including P. megasperma (P.D. Orton) Redhead, Vilgalys \& Hopple and P. schroeteri (P. Karst.) Redhead, Vilgalys \& Hopple (1.00/75). Specimens of the third species, P. ochracea nested within $P$. lilatincta complex (0.96/68). Although its position within P. lilatincta was strongly supported by Bayesian analyses, constraint analyses indicated that a monophyletic $P$. lilatincta with $P$. ochracea as a sister species cannot be rejected ( $\mathrm{p}=0.102-0.103$, au-test). Our analyses indicate the existence of further four species that we tentatively named Parasola sp. 1-4. Parasola sp. 1 forms a monophyletic group with $P$. plicatilis with strong support (1.00/97). Parasola sp. 2 groups together with P. cf. lilatincta in a weakly supported clade $(0.67 / 47)$ close to the clade which contains the true lilatincta-group. Parasola sp. 3 forms a clade with P. lilatincta and P. ochracea but this is only supported in ML analyses (199). Parasola sp. 4 forms a monophyletic group with P. misera in both the Bayesian and ML analyses $(1.00 / 98)$. 


\section{TAXONOMY}

Parasola crataegi Schmidt-Stohn, sp. nov.

FIGS. 2A, B, 3A, 4A, B

MycoBank: MB817192

Typification: GERMANY, SCHLESWIG-HOLSTEIN, Lübeck-Travemünde, Dummersdorfer Ufer (nature reserve), on grazed pasture under Crataegus monogyna, 29 Oct 2008, G. Schmidt-Stohn, SSt08-154 (holotype, M 0280274). GenBank: KY928605.

Etymology: The epithet refers to the main habitat under Crataegus.

Pileus 4-6 × 2-3 $\mathrm{mm}$ when still closed, ellipsoidal to cylindrical, then convex to hemispherical and plano-convex to applanate when expanded, up to $15 \mathrm{~mm}$ wide, gray-brown with an ochraceous-brown center when young and moist, grayish to grayish white when mature, sulcate-striate almost up to the centre. Lamellae free, up to 30 reaching the stipe at a distance of $0.5-1 \mathrm{~mm}$, additionally $15-20$ lamellules, crowded, ventricose, $<2 \mathrm{~mm}$ broad, whitish to grayish, finally blackish, with an indistinct fimbriate edge. Stipe $15-40 \times 0.5-1$ $\mathrm{mm}$, cylindrical, fistulose, translucent grayish, slightly flocculose when young, later glabrous; smell and taste not distinctive.

Basidiospores $[41,2,2] 6.5-8.5 \times 5.5-7.5 \times 4-5.5 \mu \mathrm{m}$, ave $7.4 \times 6.5 \times 4.8 \mu \mathrm{m}, \mathrm{Q}_{1}=$ $1.00-1.35, \mathrm{Q}_{1} \mathrm{av}=1.15, \mathrm{Q}_{2}=1.35-1.77, \mathrm{Q}_{2} \mathrm{av}=1.49$, rounded triangular to heart-shaped and also ovoid, flattened, ellipsoidal in lateral view, with eccentric germ-pore; Basidia in the majority (ca. 80\%) 4-spored, but some (10-20\%) also 2- or 3-spored, clavate, $31-48 \times 10-14$ $\mu \mathrm{m}$. Cheilocystidia fairly densely packed, oblong, ellipsoidal, narrowly to broadly utriform, some also spheropedunculate, $23-40 \times 10-17 \mu \mathrm{m}$. Pleurocystidia scattered, predominantly 
near the edge of lamellae, not easily detected, utriform, 48-62 $\times 17-24 \mu \mathrm{m}$. Pileipellis a hymeniderm, pileo- and sclerocystidia lacking.

Habitat: Grazed grasslands, almost always in close vicinity to single Crataegus monogyna or in scrubs of this species, basidiocarps solitary.

Distribution: Germany, Hungary.

Other specimens examined: GERMANY, SCHLESWIG-HOLSTEIN, LübeckTravemünde, Dummersdorfer Ufer (nature reserve) on grazed pasture under Crataegus monogyna, 21 Oct 1995, G. Schmidt-Stohn SSt95-113 (M 0280273); same location, 24 Oct 2009, G. Schmidt-Stohn SSt09-105 (M 0280275); SACHSEN-ANHALT, Huy, north of Halberstadt, Paulskopfwarte, on naked soil under Corylus and Crataegus, 29 Sep 1998, G. Schmidt-Stohn SSt98-239; NIEDERSACHSEN, Heeseberg west of Jerxheim on grazed pasture under Crataegus monogyna, 17 Jul 2000 SSt00-018a. - HUNGARY, BÁCSKISKUN, Fülöpháza (Kiskunság National Park), Robinia pseudoacacia plantation with Crataegus and Ailanthus, on leaf litter, 12 Oct 2008, L.G. Nagy, herb. NL-4175.

Notes: Before the description of P. crataegi, the only known species in Parasola with such small basidiospores was P. kuehneri. Therefore our new species was usually identified as $P$. kuehneri using existing keys. Considering other morphological and ecological characters, confusion of the two species is impossible: P. kuehneri has a distinctly darker pileus of $<3.5 \mathrm{~cm}$ diam and usually grows in forests and shrubs along trails on naked, mineral-, alkaline soil rather than in poorly manured grasslands like $P$. crataegi. Moreover, the basidiospores of $P$. kuehneri average $9.4 \mu \mathrm{m}$ long, while those of $P$. crataegi are only 7.4 $\mu \mathrm{m}$. Genetically, P. crataegi is closest to P. lactea (formerly P. leiocephala), forming two well separated clades. However, both the basidiospores of $P$. lactea (ave $10.7 \times 8.8 \times 6.7 \mu \mathrm{m}$ ) and the basidiocarps are much larger than those of $P$. crataegi. 
Parasola crataegi is so far known from three sites in Germany, mostly found repeatedly in different years, and one in Hungary. The habitat is almost always in grasslands/open landscape and usually in close proximity to Crataegus monogyna, as emphasized with the epithet "crataegi". Nevertheless, we suspect that P. crataegi is more widespreadbecause of confusion with P. kuehneri in the past. The only previous reference to our new species is from Bender (1989, also pers. comm.), who when commenting on Coprinus kuehneri mentioned "a deviating form under Crataegus monogyna with smaller and \pm ovoid basidiospores". His collection may be our $P$. crataegi.

Parasola ochracea L. Nagy, Szarkándi \& Dima, sp. nov. FIGS. 2/D-E, 3/B, 4/C MycoBank: MB817193

Typification: NORWAY, NORD-TRØNDELAG, Steinkjer, Skrattåsen, on cow dung, 5 Sep 2009, L.G. Nagy \& T. Knuttson, NL-3621 (holotype, BP). GenBank JN943134.

Etymology: ochracea refers to the orange to ochraceous color of the basidiocarp.

Pileus 4-20 × 3-9 $\mathrm{mm}$ when still closed, ellipsoidal to cylindrical, campanulate to flat with an umbo when expanded, up to $35(40) \mathrm{mm}$ wide, vivid orange to ochraceous, grayish orange to brownish when mature, sulcate and grooved up to the centre. Lamellae free, crowded, ventricose, $<3 \mathrm{~mm}$ broad, whitish to grayish, finally blackish, with a fimbriate edge. Stipe 30-90 × 0.5-2 mm, cylindrical, fistulose, pure white, slightly silky when young, later glabrous, smell and taste not distinctive.

Basidiospores $[40,1,1] 10-11 \times 6-8.5 \mu \mathrm{m}$, ave $10.8 \times 7.4 \mu \mathrm{m}, \mathrm{Q}=1.25-1.58, \mathrm{Qav}=$ 1.46, rounded triangular to ovoid with rounded angles, sometimes almost hexagonal, lentiform, ellipsoid in lateral view, with eccentric germ-pore. Basidia 4-spored, clavate, 35$47 \times 11-13.5 \mu \mathrm{m}$. Cheilocystidia densely packed, clavate, ellipsoidal, ovoid to balloon- 
shaped, some utriform, 42-75 $\times 25-38 \mu \mathrm{m}$. Pileipellis hymeniderm, pileo- and sclerocystidia lacking.

Habitat: On cow dung or dung mixed with straw in wet grazed meadows.

Distribution: Norway, Sweden.

Other specimens examined: SWEDEN, ÖLAND, Möckleby/Gardstorp, on cow dung and dung mixed with straw, 10 Sep 2008, L.G. Nagy \& T. Knuttson, NL-3623; ÖLAND, NedVästerstad, on cow dung, 10 Sep 2008, L.G. Nagy \& T. Knuttson, NL-3167.

Notes: Parasola ochracea is morphologically close to P. schroeteri, P. misera and P. cuniculorum, with which it shares the habitat and spore shape. However, P. schroeteri has darker colored basidiocarps and pleurocystidia, whereas $P$. misera and P. cuniculorum have much smaller basidiocarps and smaller basidiospores.

Parasola plicatilis-similis L. Nagy, Szarkándi \& Dima, sp. nov. FIGS. 2/F, 3/C, 4/D-E

MycoBank: MB817194

Typification: SWEDEN, ÖLAND, Mörbylanga parish, Södra Barspunkten, north of the road, in alvar vegetation, 28 Sep 2007, L.G. Nagy \& T. Knuttson, NL-2125 (holotype, BP). GenBank KY928620.

Etymology: similis (Latin) $=$ like, referring to the similarity of the new species to Parasola plicatilis.

Pileus 6-20 × 4-10 mm when still closed, cylindrical to ellipsoidal or ovoid, expanding to campanulate then flat, 15-45 mm when expanded, honey-colored, ochre-brown when young, becoming sordid, grayish upon ageing, with a pale ochre-brown button when 
mature, sulcate-striate up to the centre; Lamellae free, crowded, ventricose, up to $4 \mathrm{~mm}$ broad, at first whitish, then grayish and black. Stipe 40-90 × 1-2 mm, slender, fistulose, glabrous, pale ochre-brownish, slightly strigose at the base. Smell and taste not distinctive.

Basidiospores $[38,2,1] 10.5-13.5 \times 8-11.5 \mu \mathrm{m}$, ave $11.8 \times 9.7 \mu \mathrm{m}, \mathrm{Q}=1.1-1.4$, Qav $=1.2$, broadly ellipsoidal to broadly hexagonal, sometimes ovoid or almost rounded triangular, lentiform, ellipsoid in lateral view, with eccentric germ-pore. Basidia 4-spored, clavate, $30-45 \times 10-14 \mu \mathrm{m}$. Cheilocystidia utriform, clavate, sometimes broadly lageniform, $35-80 \times 12-20 \mu \mathrm{m}$, pleurocystidia utri- to lageniform, often quite narrow and slender 75-90 $\times 24-35 \mu \mathrm{m}$. Pileipellis hymeniderm, pileo- and sclerocystidia lacking.

Habitat: Grazed meadows and pastures, once found in alvar vegetation.

Distribution: Sweden, Slovakia.

Other specimens examined: SLOVAKIA, RIMAVSKÁ SOBOTA, Drňa, on grazed pasture with Festuca, 3 Oct 2008, L.G. Nagy, NL-3980. - SWEDEN, ÖLAND, Langlöt parish, $500 \mathrm{~m}$ NNW Astad, on grazed meadow, 22 Sept 2006, L.G. Nagy \& T. Knuttson, NL0287.

Notes: The species is highly similar to P. plicatilis, differing mostly in spore shape and to a smaller extent by the presence of many narrow, lageniform pleurocystidia as opposed to mostly utriform-ellipsoidal in $P$. plicatilis. The basidiospores of $P$. plicatilissimilis are mostly broadly ellipsoidal, whereas those of $P$. plicatilis are mostly ellipsoidal or hexagonal $(\mathrm{Q}=1.15-1.5$ vs. $1.25-1.6)$. The spore shape of $P$. plicatilis-similis is transitional between that of earlier diverging ellipsoid-spored species ( $P$. plicatilis, P. auricoma) and more derived species with rounded triangular basidiospores (e.g. P. schroeteri, P. lactea). Accordingly, the species present a continuum in spore shape and their identification should 
rely on the shape of the majority of basidiospores and measurements of 10-20 individual basidiospores.

\section{KEY TO DESCRIBED AND UNDESCRIBED}

\section{SPECIES OF PARASOLA.}

1. Pileus with long brown, thick-walled hairs ("sclerocystidia")

1.' Pileus without sclerocystidia .5

2. Basidiocarp psathyrelloid, pileus not sulcate, not deliquescent, does not collapse when mature

P. conopilus

2.' Basidiocarp collapses when mature

3a Basidiospores ellipsoidal to oblong

P. auricoma

3.' Basidiospores rounded triangular 4

4. Basidiospores subglobose to broadly ovoid, $8-11 \mu \mathrm{m}$ long

P. setulosa

4.' Basidiospores broadly ellipsoidal to subglobose, $11-18 \mu \mathrm{m}$ long P. malakandensis

5. Pleurocystidia absent, exclusively coprophilous 6

5.' Pleurocystidia present, can occur on dung .8

6. Basidiocarps large, pileus $>20 \mathrm{~mm}$ when expanded P. ochracea

6.' Basidiocarps smaller .7

7. Basidia 4-spored P. misera

7.' Basidia 2-spored P. cuniculorum

8. Average spore length 7-9.5 $\mu \mathrm{m}$ 9

8.' Average spore length longer 10

9. Average spore length $9.5 \mu \mathrm{m}$, pileus dark brown to red-brown, expanded up to $35 \mathrm{~mm}$ wide, in forests or along trails P. kuehneri 
9.'Average spore length $7.5 \mu \mathrm{m}$, pileus gray-brown, grayish to grayish white, expanded up to $15 \mathrm{~mm}$ wide, in grasslands mostly under Crataegus P. crataegi

10. Basidiospores ave 9-13 $\mu \mathrm{m}$ long .11

10.' Basidiospores ave longer than $12.5 \mu \mathrm{m}$ 15

11. Basidiospores with germ pore, central or eccentric to the adaxial side, basidiospores often broader than long

Parasola sp. 2

11.' Germ pore eccentric to the opposite side of the hilum (abaxial side) 12

12. Basidiospores narrowly ovoid to ellipsoidal, sometimes ovoid, Qav $=1.15-1.60$ 13

12.' Basidiospores ovoid, rounded triangular or subglobose 14

13. Basidiospores mostly ellipsoidal to hexagonal, $\mathrm{Qav}=1.15-1.5$, pleurocystidia mostly utriform P. plicatilis

13.' Basidiospores broadly ellipsoidal, often ovoid to broadly hexagonal, Qav $=1.25-1.6$, pleurocystidia often lageniform P. plicatilis-similis

14. Average length of basidiospores under $10.5-11 \mu \mathrm{m}$, basidiospores often remain immature P. lactea (= P. leiocephala)

14.' Average length of basidiospores $>11.5 \mu \mathrm{m}$, as usual fully mature, dark blackish brown, pileipellis and basidia filled with strongly refringent, yellowish granules

P. lilatincta s.l.

(if granules absent and basidiospores ave $>11 \mu \mathrm{m}$ long: see $\boldsymbol{P}$. schroeteri)

15. Basidiospores narrowly ovoid to ellipsoidal, 14-19 $\mu \mathrm{m}$ long P. megasperma (if basidiospores smaller, $12-14 \mu \mathrm{m}$, see $\boldsymbol{P}$. plicatilis)

15.' Basidiospores ovoid, rounded triangular to subglobose .16

16. Basidiospores $12-14 \mu \mathrm{m}$ long P. schroeteri

16.' Basidiospores 13-18 $\mu \mathrm{m}$ long P. hercules (plus $\boldsymbol{P}$. aff. hercules and other undescribed species) 


\section{DISCUSSION}

In this study we describe three new species, Parasola crataegi, $P$. ochracea and P. plicatilissimilis. All were collected in central Europe (Germany, Hungary) or northern Europe (Norway, Sweden), but wider geographical distributions are highly likely given their relationships to well-known species with broader documented distribution. All three species prefer grassland habitats, especially lawns or marginal grass patches close to forest trails.

Parasola crataegi is morphologically similar to P. kuehneri, but has significantly smaller basidiospores and basidiocarps, a different habitat and is genetically more closely related to $P$. lactea.

Parasola ochracea is phylogenetically closest to $P$. lilatincta, although morphologically it is more similar to $P$. schroeteri and P. misera. While all three species are more or less coprophilous, the conspicuous ochraceous to orange color of the pileus distinguishes $P$. ochracea from $P$. schroeteri, while $P$. misera has significantly smaller basidiocarps and differently shaped basidiospores. In the ML and Bayesian analyses $P$. ochracea formed a species-level lineage nested within a species complex around P. lilatincta. However, both morphological characters and constraint analyses suggest that $P$. ochracea is distinct from P. lilatincta.

Parasola plicatilis-similis is morphologically similar to $P$. plicatilis and forms a monophyletic group with the $P$. schroeteri-P. megasperma clade, whereas $P$. plicatilis branches off earlier in the crown-group Parasola. Parasola plicatilis-similis differs from $P$. plicatilis in having wider and differently shaped basidiospores and the habitat on dung. It is noteworthy that a significant intraspecific variability in stipe coloration was observed in $P$. plicatilis. We consistently encounter specimens with lilaceous to brown stipes, which 
generally have larger basidiocarps than typical $P$. plicatilis specimens, although no evidence for the genetic separation of these specimens from typical $P$. plicatilis specimens was found in the genes used in this study.

We also found evidence for further undescribed species, but these lack sufficient specimen information and/or molecular data to allow them to be described in this study (Parasola sp. 1-4). These results and other recent reports of new species i.e. Parasola cuniculorum (Schafer 2014) and P. malakandensis (Hussain et al. 2017), suggest a considerable undescribed diversity in Parasola and probably also in other coprinoid lineages of Psathyrellaceae.

\title{
ACKNOWLEDGEMENTS
}

The authors thank Jan Červenka for providing specimens for this study. This work was funded by the grant OTKA NN106394 and the infrastructural project GINOP-2.3.3-15-201600006. LGN and TP are granted by LP2014/12 and LP2016-8/2016, respectively.

\section{LITERATURE CITED}

\begin{abstract}
Altschul SF, Gish W, Miller W, Myers EW, Lipman DJ. 1990. Basic local alignment search tool. Journal of Molecular Biology 215: 403-410.
\end{abstract}

Buller AHR. 1931. Researches on the Fungi IV: Further observations on the Coprini together with some investigations on social organization and sex in the 'Hymenomycetes'. London, New York: Longmans Green \& Co. 329 p.

Bender H. 1989. Gattung Coprinus Sektion Pseudocoprinus. Beschreibung und Gegenüberstellung der Arten Coprinus leiocephalus und Coprinus kuehneri. APN. Mitteilungsblatt der Arbeitsgemeinschaft Pilzkunde Niederrhein 7: 36-45. 
Gardes M, Bruns TD. 1993. ITS primers with enhanced specificity for basidiomycetesapplication to the identification of mycorrhizae and rusts. Molecular Ecology 2: 113-118.

Hussain S, Afshan NS, Ahmad H, Khalid AN, Niazi AR. 2017. Parasola malakandensis sp. nov. (Psathyrellaceae; Basidiomycota) from Malakand, Pakistan. Mycoscience 58: 69-76.

Larsson E, Örstadius L. 2008. Fourteen coprophilous species of Psathyrella identified in the Nordic countries using morphology and nuclear rDNA sequence data. Mycological Research 112: 1165-1185.

Löytynoja A, Goldman N. 2005. An algorithm for progressive multiple alignment of sequences with insertions. Proceedings of the National Academy of Sciences USA 102: $10557-10562$.

Maddison WP, Maddison DR. 2011. Mesquite: a modular system for evolutionary analysis.

version 2.75. http://mesquiteproject.org.

Nagy LG, Kocsubé S, Papp T, Vágvölgyi, C. 2009. Phylogeny and character evolution of the coprinoid mushroom genus Parasola as inferred from LSU and ITS nrDNA sequence data. Persoonia 22: $28-37$

Nagy LG, Vágvölgyi C, Papp T. 2010a. Type studies and nomenclatural revisions in Parasola (Psathyrellaceae) and related taxa. Mycotaxon 112: 103-141.

Nagy LG, Urban A, Örstadius L, Papp T, Larsson E, Vágvölgyi C. 2010b. The evolution of autodigestion in the mushroom family Psathyrellaceae (Agaricales) inferred from Maximum Likelihood and Bayesian methods. Molecular Phylogenetics and Evolution 57: 1037-1048.

Nagy LG, Vágvölgyi C, Papp T. 2012. Morphological characterization of clades of the Psathyrellaceae (Agaricales) inferred from a multigene phylogeny. Mycological Progress 12: $505-517$. 
Padamsee M, Matheny PB, Dentinger BTM, Mclaughlin DJ. 2008. The mushroom family Psathyrellaceae: evidence for large-scale polyphyly of the genus Psathyrella. Molecular Phylogenetics and Evolution 46: 415-429.

Rambaut A, Suchard MA, Xie D, Drummond AJ. 2014. Tracer v 1.6. Tracer available online at http://beast.bio.ed.ac.uk/Tracer

Redhead SA, Vilgalys R, Moncalvo JM, Johnson J, Hopple JS Jr. 2001. Coprinus Persoon and the disposition of Coprinus species sensu lato. Taxon 50: 203-241.

Ronquist F, Teslenko M, van der Mark P, Ayres DL, Darling A, Höhna S, Larget B, Liu L, Suchard MA, Huelsenbeck JP. 2012. MrBayes 3.2: Efficient Bayesian phylogenetic inference and model choice across a large model space. Systematic Biology 61: 539-542.

Schafer DJ. 2010. Keys to sections of Parasola, Coprinellus, Coprinopsis and Coprinus in Britain. Field Mycology 11: 44-51.

Schafer DJ. 2014. The genus Parasola in Britain, including Parasola cuniculorum sp. nov. Field Mycology 15: 77-99.

Schmidt-Stohn G. 2012. Drei seltene Tintlinge - Untersuchungsmethoden, Merkmale, taxonomische Stellung und Verbreitung in Europa. Zeitschrift für Mykologie 78: 137-153.

Shimodaira H, Hasegawa M. 2001. CONSEL: for assessing the confidence of phylogenetic tree selection. Bioinformatics 17: 1246-1247.

Simmons MP, Ochoterena H. 2000. Gaps as characters in sequence-based phylogenetic analyses. Systematic Biology 49: 369-382.

Stamatakis A. 2006. Maximum likelihood-based phylogenetic analyses with thousands of taxa and mixed models. Bioinformatics 22: 2688-2690. 
Sukumaran J, Holder MT. (2010) DendroPy: A Python library for phylogenetic computing. Bioinformatics 26: 1569-1571.

Waterhouse AM, Procter JB, Martin DMA, Clamp M, Barton GJ. 2009. Jalview Version 2-a multiple sequence alignment editor and analysis workbench. Bioinformatics 25: 1189-1191.

White TJ, Bruns T, Lee S, Taylor J. 1990. Amplification and direct sequencing of fungal ribosomal RNA genes for phylogenetics. In: Innis MA, Gelfand DH, Sninsky JJ, White TJ, eds. PCR Protocols: a guide to methods and applications. New York, NY: Academic Press. p. $315-322$. 
Figure 1. 50\% majority rule phylogram constructed from post-burn-in trees of the Bayesian analyses of $28 \mathrm{~S}+\mathrm{ITS}$ data. Numbers on the branches represent Bayesian posterior probabilities followed by ML bootstrap values. An asterisk (*) indicates $100 \%$ bootstrap or 1.00 posterior probablities while a minus sign $(-)$ indicates support values lower than $50 \%$ boostrap or 0.70 posterior probability. Sequences of the newly described species highlighted in boldface. Bar indicates 0.01 expected change per site per branch. 


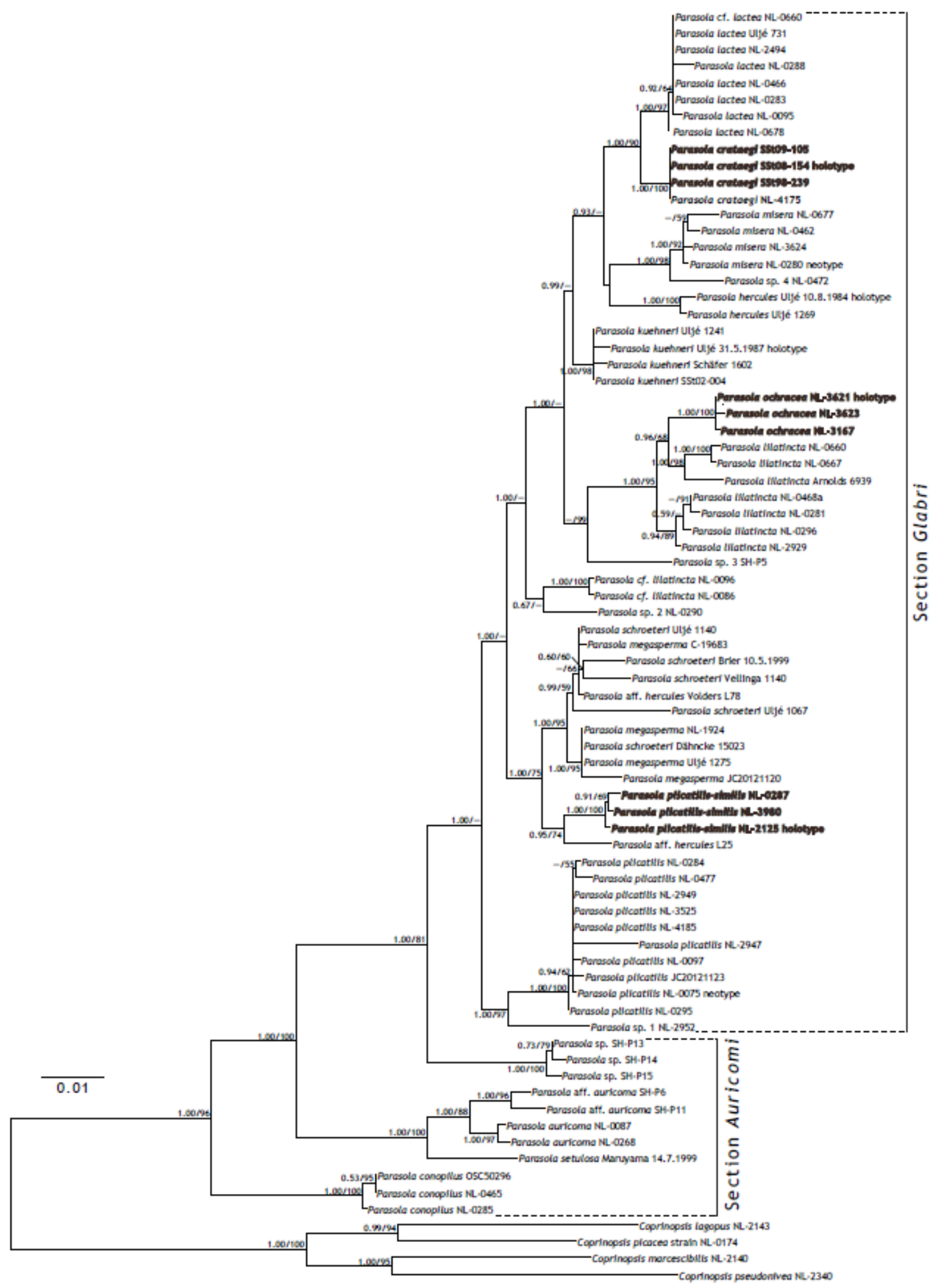


Figure 2. Macromorphology of P. kuehneri and the newly described Parasola species. A, B: P. crataegi, C, D: P. kuehneri, E, F: P. ochracea, G: P. plicatilis-similis. Photos: A-D: G. Schmidt-Stohn; E, G: L. Nagy. Bars $=1 \mathrm{~cm}$.
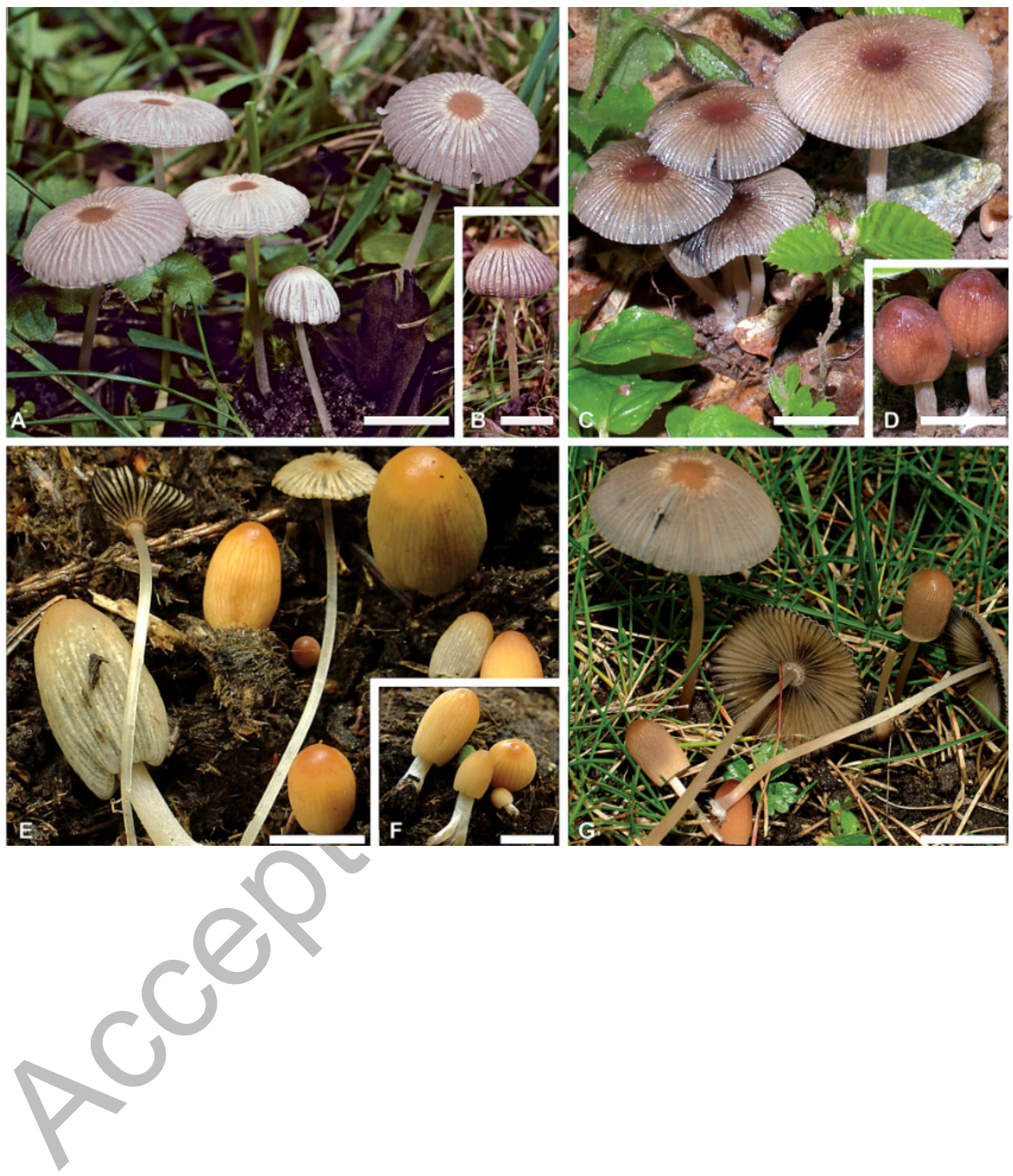
Figure 3. Illustration of the diversity of basidiospore shapes and sizes in the newly described species. A. Parasola crataegi. B. P. ochracea. C. P. plicatilis-similis.

A<smiles>c1ccccc1</smiles>

B<smiles>C1CCCCCC1</smiles>

$\mathrm{C}$

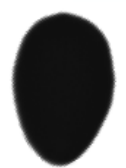<smiles>c1ccccc1</smiles><smiles>C1CCCCC1</smiles>
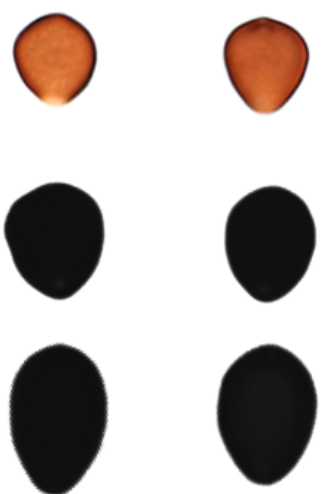
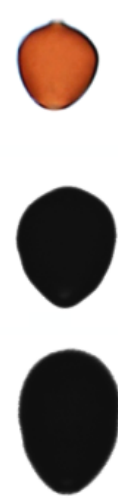
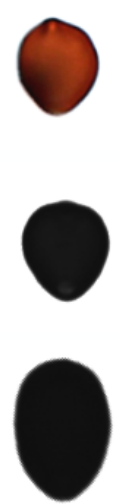
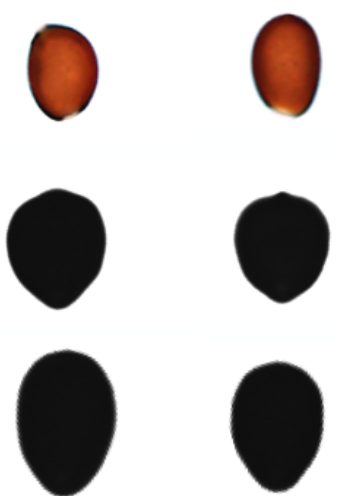

$10 \mu \mathrm{m}$ 
Figure 4. Cheilo- and pleurocystidial characters of Parasola species. A, B. P. crataegi. A. Cheilocystidia. B: Pleurocystidia. C. P. ochracea cheilocystidia. D, E. P. plicatilis-similis. D. Pleurocystidia. E: Cheilocystidia.

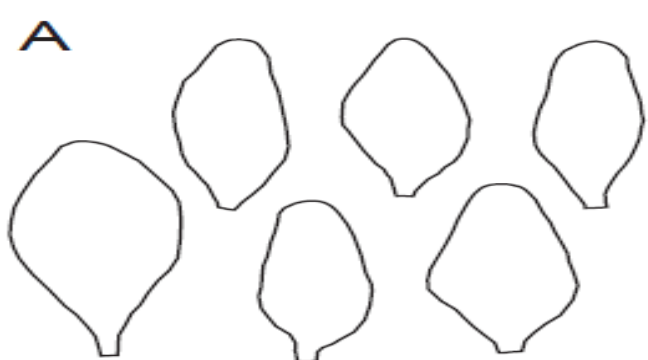

B
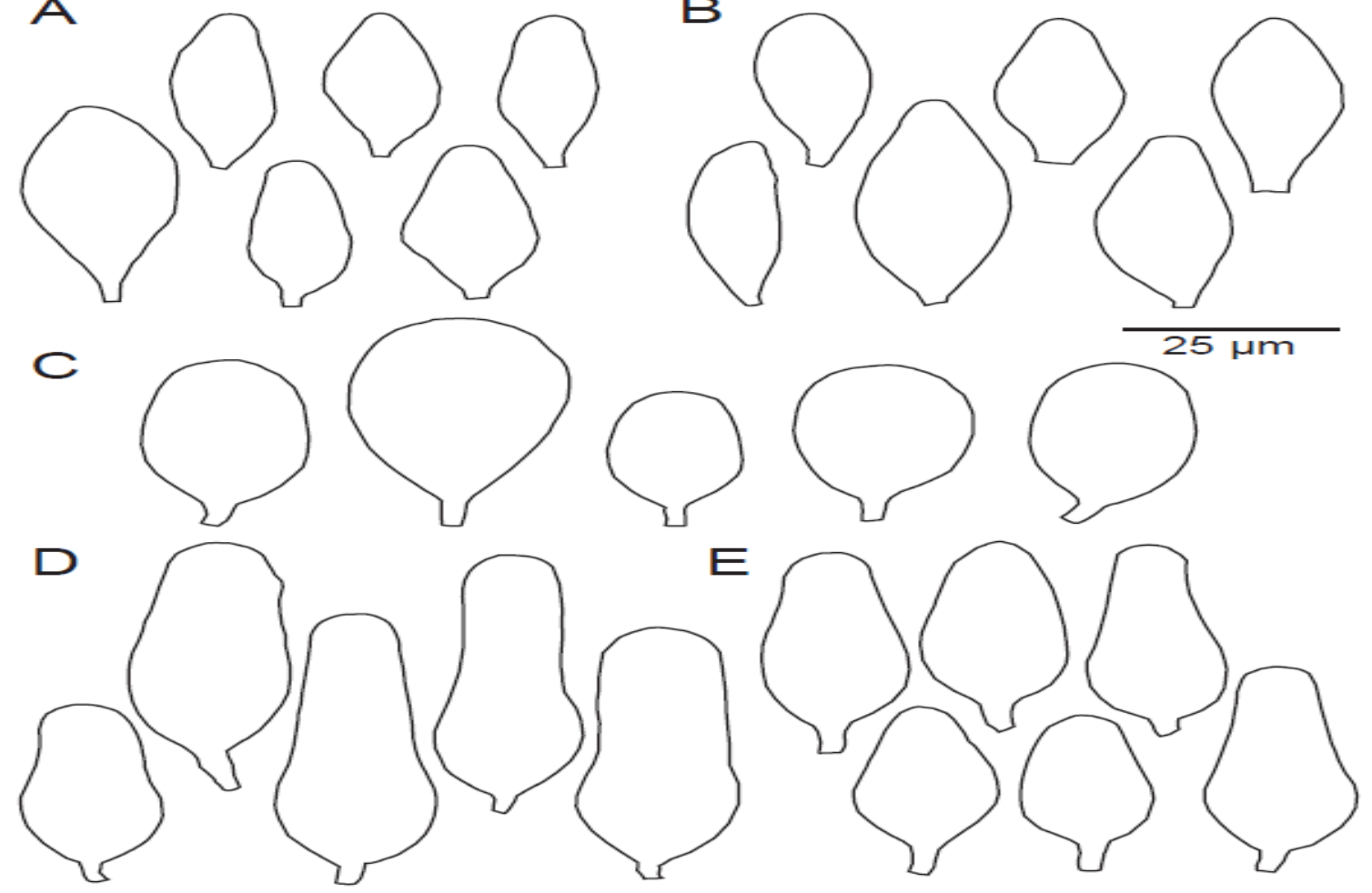